Mitteilungen aus dem pharm.-chem. Laboratorium zu Braunschweig.

Von H. Beckurts.

\title{
Zur quantitativen Bestimmung des Zuckers in diabetischem Harn.
}

\author{
Von J. Troeger und W. Meine.
}

Diabetischer Harn bereitet zuweilen bei der quantitativen Bestimmung des Zuckers insofern Schwierigkeiten, als das Kupferoxydulbezw. hydrat, welches aus dem in der Fehling'schen Lösung enthaltenen Kupferoxyd durch Reduktion gebildet wird in der Flüssigkeit so fein verteilt ist, dass ein Absitzenlassen oder Filtrieren unmöglich wird. Derartige Erscheinungen sind wiederholt beobachtet worden und sucht man sie dadurch zu erklären, dass gewisse in dem menschlichen Körper gebildete und vor allem im Harn enthaltene physiologische Stoffe die Abscheidung des Kupferhydroxyduls verhindern. Die Litteratur giebt hierfür reichlich Belege und führt auch Mittel an, die in solchen abnormen Fällen ein rasches Absitzen des Kupteroxyduls bewirken sollen. Die angegebenen Methoden, wie Schütteln mit einigen Tropfen konzentrierter Chlorcalciumlösung, mit Filtrierpapier oder auch schnelles Abkühlen der mit dem Harn gekochten Fehling'schen Lösung führten jedoch bei den von uns speziell untersuchten Proben des diabetischen Harns nicht zum Ziele. Ein schnelles Absitzen ist deshalb notwendig, weil sonst bereits abgeschiedenes Kupferoxydul beim Erkalten wieder in Lösung geht, was eine richtige Bestimmung von vorn herein natürlich ausschliesst. Als daher in solchem abnormen Harn der Zuckergehalt bestimmt werden sollte, liess die übliche Titriermethode vollständig im Stich, selbst als eine bestimmte Menge Zucker zur Erhöhung des Zuckergehaltes hinzugefügt wurde, konnte eine quantitative Bestimmung desselben nicht ausgeführt werden. Der Grund hierfür lag darin, dass bei noch so grossem Verbrauch des diabetischen Harns stets Kupfer in Lösung blieb. Die angestellte Tüpfelreaktion, die behufs Zurückhaltung der suspendierten Oxydulteilchen derart ausgeführt wurde, dass man einen Tropfen der zu prüfenden Flüssigkeit auf einen über das Ferrocyankaliumpapier gelegten Filtrierpapierstreifen bringt, liess immer noch eine Färbung, die von Kupfer herrührte, deutlich erkennen. Auffallend war, dass diabetischer Harn, von demselben Kranken herrührend, bei den ersten Untersuchungen, als der Zuckergehalt nahezu 7\% betrug, diese Erscheinungen nicht zeigte. Das Schmi dt'sche 
Verfahren (vergl. Schmidt, pharm. Chemie Bd. II. (2. Auflage), Seite 754), bei dem man eine gewisse Menge Fehling'scher Lösung mit wechselnden Mengen Harn erhitzt, konnte aus dem oben erwähnten Grunde gleichfalls nicht angewandt werden, ebenso die Allihn'sche Methode, bei der infolge des langsamen Filtrierens immer ein Teil Kupfer wieder in Lösung ging. Es blieben somit nur das polarimetrische Verfahren, sowie die Gärungsmethoden übrig.

Polarimetrisch liess sich der Zuckergehalt des Harns allerdings in einigen Fällen bestimmen, doch machte sich meist eine Klärung des Harns notwendig. In diesem speziellen Falle wurde dieselbe dadurch erreicht, dass der Harn zunächst mit einem abgemessenem Volumen Bleiacetatlösung geschüttelt wurde, worauf das Blei durch Behandeln des Harns mit Zinkstaub entfernt wurde und so eine sehr klare Flüssigkeit resultierte. Da einerseits jedoch einem jeden Experten ein Polarisationsapparat nicht immer zur Verfügung steht, andrerseits bei geringen Zuckermengen, zumal bei Anwesenheit von unbekannten physiologischen Stoffen, auch die Resultate etwas fragwürdig erscheinen dürften, so ist diese Methode nicht immer anwendbar oder unter Umständen nicht genügend zuverlässig.

Was die Gärungsmethoden schliesslich anbelangt, so können die hierbei erzielten Werte nur annähernde sein, doch ist aus den weiter angeführten, durch praktische Versuche festgestellten Beispielen ersichtlich, dass in derartigen Fällen, wo ein Polarisationsapparat nicht zur Verfügung steht, immerhin annehmbare Resultate sich ergeben, die für praktische Zwecke, namentlich für fortlaufende Bestimmungen recht brauchbar sind. Dies letztere ist speziell von dem Einhorn'schen Saccharimeter zu sagen, das aus zwei kommunizierenden Röhren besteht, deren eine kugelig aufgeblasen ist und das mit $10 \mathrm{ccm}$ des $\mathrm{zu}$ untersuchenden Harns, sowie etwas Hefe und einer Spur Weinsäure, die die Gärung schneller einleitet, beschickt wird. Bei einer Temperatur von $35-40^{\circ} \mathrm{C}$. beginnt die Umsetzung des Zuckers in Alkohol und Kohlensäure, welch letztere in dem empirisch kalibrierten Apparate aufgetangen wird. Da dieser Apparat nur Zucker bis $1 \%$ anzeigt, so ist der Harn je nach dem spez. Gewicht des Harns vorher in entsprechender Weise zu verdünnen. Im Interesse der Genauigkeit bei dieser Methode sei noch erwähnt, dass das Befreien des Harns von atmosph. Luft durch vorheriges Auskochen sehr zu empfehlen ist, da sonst die durch Gärung gefundenen Werte sich meist als zu hoch herausstellen. Das Ablesen der entwickelten Kohlensäuremenge muss bei einer Temperatur von $20^{\circ} \mathrm{C}$. geschehen. Ein anderes Verfahren gründet sich auf die Bestimmung des spez. Gewichts des Harns vor und nach Ablauf einer eingeleiteten 
alkoholischen Gärung. Die Differenz mit einem hier nicht näher abzuleitenden Faktor multipliziert, giebt direkt den Prozentgehalt an. Der Nachteil bei diesem Verfahren liegt darin, dass der Faktor schwankend und relativ gross ist, sodass ein verhältnismässig recht geringer Fehler bei Feststellung der spez. Gewichte durch die Multiplikation erheblich vergrössert wird. Die spez. Gew. müssen ausserdem mittelst eines möglichst grossen Pyknometers recht genau ermittelt werden.

Ein weiteres Gärungs-Saccharimeter ist der Gutzeit'sche Apparat. Derselbe beruht auf demselben Prinzip wie der Einh orn'sche, nur, dass hier die gesamte Kohlensäuremenge über Quecksilber aufgefangen wird und man dann den Zuckergehalt an einer empirisch festgestellten Skala, die bis 10\% Zucker anzeigt, abliest. Da hierbei eine sehr minimale Harnmenge $(0,5 \mathrm{ccm})$ zur Bestimmung nur verwandt wird, so ist es notwendig, dass diese auf das Genaueste abgemessen wird. Die Gärung ist ziemlich schnell beendet, während die Genauigkeit absolut keine grössere ist, wie bei den vorhin angeführten Gärungsmethoden.

Da nun, wie eingangs der Abhandlung erwähnt, auf Zucker zu prüfende Harne vorkommen können, bei denen die üblichen gewichtsanalytischen Methoden aus den schon oben angeführten Gründen nicht brauchbar sind, so sind wir bisher nicht im Stande, solche Harne auf Grund einer zuverlässigen Methode auf ihren Zuckergehalt zu untersuchen. Die Ergebnisse der Polarisation und Gärungsmethoden können zwar recht zuverlässige sein, müssen jedoch bei anormalem Harn mit grosser Vorsicht aufgenommen werden, so lange es nicht möglich ist, die Polarisation und die Gärungsmethoden durch irgend eine andere gewichtsanalytische Methode zu kontrollieren. Aus diesem Grunde wurde versucht, ein Verfahren auszuarbeiten, welches es ermöglicht, bei anormalem diabetischen Harn den Zuckergehalt desselben auch dann zu bestimmen, wenn solch diabetischer Harn mit Fehling'scher Lösung gekocht, das Kupferoxydul in sehr fein verteiltem Zustand in der Lösung enthält. Nach folgender Methode gelang es schliesslich nach vielen vergeblichen Versuchen, den Zuckergehalt in solch anormalem Harne zu ermitteln. Verwendet wurde eine modifizierte $\mathrm{F}$ ehling'sche Lösung die anstatt der vorgeschriebenen $173 \mathrm{~g}$ Seignettesalz $100 \mathrm{~g}$ Glyzerin im Liter enthielt. Der Kupfergehalt dieser Lösung wurde gewichtsanalytisch ermittelt und zwar in der Weise, dass in einem aliquoten Teil der Lösung nach dem Ansäuern derselben das Kupfer in Form von Halbschwefelkupfer bestimmt wurde. Der Ersatz des Seignettesalzes durch Glyzerin ist aus dem Grunde nötig, weil die Säure eine Abscheidung von Weinsteinkrystallen bewirkt und diese durch Auswaschen von dem Schwefelkupferniederschlag nicht zu trennen sind. 
Zur quantitativen Bestimmung des Zuckers im Harn wurden jedesmal $60 \mathrm{ccm}$ der glyzerinhaltigen Kupferlösung verwendet, welche in einer Porzellanschale mit 5-20 ccm Harn (je nach dem Zuckergehalt) 15 Minuten lang erhitzt wurden. Der heisse Schaleninhalt wurde alsdann in einen Mafskolben $(100 \mathrm{ccm})$ gespült und aufgefüllt bis zur Marke, die für die Temperatur $45^{\circ} \mathrm{C}$. eingestellt war. Diese Mischungstemperatur von $45^{\circ} \mathrm{C}$, auf welche die korrigierte Marke des Kolbens Bezug hat, wurde durch eine Reihe von empirischen Versuchen ermittelt. Der warme Inhalt des Kolbens, der das Kupferoxydul in sehr fein verteilter Form suspendiert enthält, wird hierauf mit Talkum kräftig durchgeschüttelt und dann, um ein schnelles Filtriren zu ermöglichen, gleichzeitig durch zwei dichte Filter filtriert. Ein Teil des erkalteten Filtrats wurde abgemessen, hierin der Kupfergehalt in der oben beschriebenen Weise bestimmt und schliesslich auf die Gesamtmenge, $100 \mathrm{ccm}$, umgerechnet. Zieht man nun diesen Kupfergehalt von der in den angewandten $60 \mathrm{ccm}$ enthalten gewesenen Kupfermenge $a b$, so erhält man diejenige Kupfermenge, welche von dem Harnzucker reduziert worden ist. Mit Hilfe der Allihn'schen Tabellen lässt sich leicht die in dem abgemessenen Volum Harn enthaltene Zuckermenge und somit der Prozentgehalt des Harnes an Zuker feststellen.

In nachstehender Tabelle sind die verschiedenen Werte verzeichnet, welche bei der Bestimmung des Zuckers in diabetischem Harn nach der eben beschriebenen gewichtsanalytischen Methode, sowie der Polarisationsmethode und den Gärungsmethoden erhalten worden sind.

$\begin{array}{ccccc} & \begin{array}{c}\text { Gewichtsanalytische } \\ \text { Methode }\end{array} & \begin{array}{c}\text { Gärungsmethode } \\ \text { nach Einhorn }\end{array} & \begin{array}{c}\text { Gärungsmethode } \\ \text { (Diff. d. spez. Gew.) }\end{array} & \text { Polarisationsmethode } \\ \text { m. mod. Fehl. Lösung } & \mathbf{1 , 3 6} & \mathbf{1 , 4 0} & \mathbf{1 , 3 4} & 1,41 \\ \text { I. } & 0,86 & 0,89 & 0,87 & 0,90 \\ \text { II. } & 0,86 & 0,79 & - & - \\ \text { III. } & 0,79 & 0,63 & - & - \\ \text { IV. } & 0,60 & & \end{array}$

Zur weiteren Kontrolle der in der vorstehenden Abhandlung beschriebenen Methode wurde in Zuckerlösungen von bekanntem Gehalt der Zucker mittelst der neuen gewichtsanalytischen Methode und gleichzeitig nach den beiden angeführten Gärungsmethoden bestimmt.

Nachstehende Tabelle giebt eine Uebersicht der an verschiedenen Proben erhaltenen Werte.

$\begin{array}{rcccc}\text { PCt.-Geh. d. wäss. } & \begin{array}{c}\text { Gewichtsanalyt. } \\ \text { Methode }\end{array} & \text { Einhorn } & \text { Pyknometer } \\ \text { I. } & 1,0025 & 0,9985 \% & 1,00 \% & 1,012 \% \\ \text { II. } & 0,9987 & 1,0015 \% & 0,98 \% & - \\ \text { III. } & 0,8783 & 0,869 \% & - & -\end{array}$


J. Troeger u. E. Ewers: Alkylthiosulfonierte Acetessigester. $\quad 309$

Schliesslich wurden normale Harne noch mit wechselnden Mengen Traubenzuckers versetzt und analysiert.

In nachstehender Tabelle sind die gefundenen Werte verzeichnet.

$\begin{array}{rccc} & \text { pCt. Zucker d. Harns } & \begin{array}{c}\text { Gewichtsanalytische } \\ \text { Methode }\end{array} & \text { Einh or n } \\ \text { I. } & 0,2370 & 0,2395 & 0,25 \\ \text { II. } & 0,9528 & 0,9530 & 0,96 \\ \text { III. } & 2,9850 & 2,9880 & 3,1 .\end{array}$

\section{Ueber alkylthiosulfonierte Acetessigester.}

Von J. Troeger und E. Ewers.

Wie bereits $R$. Otto und A. Rössing ${ }^{1}$ ) nachgewiesen haben, lässt sich durch Einwirkung von benzolsulfinsaurem Natrium auf Chloracetessigester nicht der phenylsulfonierte Acetessigester darstellen, sondern es resultiert dabei nur Phenylsulfonessigsäureester, während die saure Reaktion der Reaktionsflüssigkeit nach beendeter Umsetzung deutlich für eine Abspaltung der Acetylgruppe aus dem Acetessigester spricht. R. Otto und A. Rössing erhielten bei ihren Versuchen den Phenylsulfonessigsäureester, der aus Aether in gut ausgebildeten Prismen vom Smp. 41-42 ${ }^{\circ}$ gewonnen wurde. Als wir diese Versuche wiederholten, zeigte sich, dass bei Einwirkung von sulfinsauren Salzen auf Chloracetessigester unter Sauerwerden der Reaktionsflüssigkeit sich nur sulfonierte Essigester bilden. So lieferte beispielsweise benzolsulfinsaures $\mathrm{Salz}$ und Chloracetessigester den von Otto beschriebenen Phenylsulfonessigester, dessen Schmelzpunkt sich bei ca. $43^{\circ}$ ergab und der bei der Analyse 13,94\% $\mathrm{S}$ lieferte, entsprechend der Formel $\mathrm{CH}_{2}\left(\mathrm{SO}_{2} \mathrm{C}_{6} \mathrm{H}_{5}\right) \mathrm{COOCO}_{2} \mathrm{H}_{5}$, welche $14,04 \% \mathrm{~S}$ verlangt, während ein phenylsulfonierter Acetessigester $\mathrm{CH}_{3} \mathrm{CO} . \mathrm{CH}$ $\left(\mathrm{SO}_{2} \mathrm{C}_{6} \mathrm{H}_{5}\right) . \mathrm{COO} \mathrm{C}_{2} \mathrm{H}_{5}$ einen $\mathrm{Sch}$ wefelgehalt von $11,8 \%$ fordert. Wie die oben zitierte Abhandlung zeigt, blieben auch alle anderen Versuche, die R. Otto und A. Rössing anstellten, um zu einem sulfonierten Acetessigester zu gelangen, ohne Erfolg. In der Litteratur liegen weitere Versuche vor, die bezwecken, durch Einwirkung von Natriummercaptid auf Chloracetessigester zu einem Thiophenylacetessigester zu gelangen. Sowohl die Versuche von A. Hantzsch ${ }^{2}$ ) als auch von

1) R. Otto und A. Rössing, Ber. 23, 752.

2) A. Hantzsch, Ber. 19, 1297. 